\title{
Dynamic Stability Testing of the Mars Science Laboratory Entry Capsule
}

\author{
Mark Schoenenberger, NASA Langley Research Center, Hampton VA \\ Leslie Yates`, AerospaceComputing Inc., Mountain View, CA \\ Wayne Hathaway ${ }^{\ddagger}$ Arrow Tech Associates, So. Burlington, VT
}

\begin{abstract}
Results from a 26 shot ballistic range test of the Mars Science Laboratory (MSL) entry capsule are presented. The supersonic pitch damping properties of the MSL capsule were characterized between Mach 1.35 and Mach 3.5 and total angles-of-attack from $0^{\circ}$ to $30^{\circ}$. In flight, the MSL entry capsule will utilize a radial center-of-gravity offset to produce a non-zero trim angle-of-attack. This offset trim angle will produce lift, enabling the capsule to fly a guided entry and reducing the landing footprint dimensions to within $10 \mathrm{~km}$ of the desired landing site. A lifting configuration could not be tested at the ballistic range used for this test as the models would swerve into the range walls, possibly damaging cameras, the coordinate reference system or other facility assets. Ballistic (non-lifting) data was extracted and will be implemented in a conservative fashion to ensure that the dynamic stability characteristics of the flight vehicle are bounded. A comparison between the MSL pitch damping results and the dynamic model of the Mars Exploration Rover capsule shows generally close agreement with no significant differences in damping characteristics due to the change in backshell geometry. Dynamic moments are also compared to the MSL reaction control system (RCS) control authority to show the controller has sufficient margin to easily damp any dynamic stability effects.
\end{abstract}

*Aerospace Engineer, Atmospheric Flight and Entry Systems Branch, Member AIAA

$\dagger$ Vice President, Senior Member AIAA

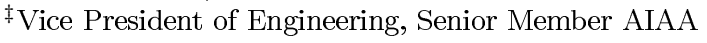




\section{Nomenclature}

$\begin{array}{llll}A & \text { Amplitude constant } & \nu & \text { Euler-Cauchy frequency } \\ C_{A} & \text { Axial force coefficient } & \xi & \text { Amplitude growth constant } \\ C_{L_{\alpha}} & \text { Lift slope coefficient } & \rho & \text { Density } \\ C_{m_{\alpha}} & \text { Pitching moment slope coefficient. } & \theta, \psi & \text { Euler angles } \\ C_{m_{q}} & \text { Pitch damping coefficient } \frac{\partial C_{m}}{\partial\left(\frac{q d}{2 V}\right)}+\frac{\partial C_{m}}{\partial\left(\frac{\dot{\alpha}}{2 V}\right)} & \omega & \text { Oscillation frequency } \\ \overline{C_{m_{q}}} & \text { Effective (averaged) pitch damping } & \text { Subscripts } & \\ C_{n_{r}} & \text { Yaw damping coefficient } & C E & \text { Constant energy } \\ d & \text { Diameter } & c g & \text { Center of gravity } \\ I & \text { Moment of inertia } & o & \text { Peak amplitude } \\ M & \text { Mach } & q & \text { Pitch rate } \\ m & \text { Mass } & r e f & \text { Reference value } \\ S & \text { Reference Area } & T & \text { Total } \\ T & \text { Torque } & T r i m & \text { Trim angle of attack } \\ U & \text { Uncertainty } & x x, y y, z z & \text { Moment of inertia axes } \\ V & \text { Velocity } & \infty & \text { Freestream } \\ x, y, z & \text { Body coordinate axes } & \text { Superscripts } & \\ \text { Greek } & & \text { A } & \text { Adder } \\ \alpha & \text { Angle of attack } & \mathrm{M} & \text { Multiplier } \\ \delta & \text { Phase shift constant } & & \\ \epsilon & \text { Sine of total angle } & & \\ \mu & \text { Amplitude growth exponent } & & \end{array}$

\section{Introduction}

The Mars Science Laboratory will land on Mars in 2012. The 4.5m diameter entry vehicle (EV) will be the largest flown to Mars, carrying the largest payload, a $900 \mathrm{~kg}$ rover. The MSL capsule geometry is similar to other recent Mars entry vehicles. Each has been based on the original $70^{\circ}$ sphere-cone Viking capsules which landed in the 1970's. Mars Pathfinder changed the backshell shape to accommodate the payload packaging. The Pathfinder shape was used by the Mars Exploration Rovers and Mars Phoenix, but MSL has again changed the backshell to accommodate the payload. Blunt bodies going back to Viking and earlier have found dynamic instabilities that arise as the capsules decelerate to Mach 3.5 and below. ${ }^{1}$ These instabilities are thought to be driven by a wake pressure lag as the capsule oscillates. ${ }^{2}$ Therefore, the dynamic stability characteristics of blunt bodies can be very dependent on backshell geometry. Prior experience with the backshell designs of the Stardust and Genesis entry capsules ${ }^{3,4}$ demonstrated that small modifications can produce large changes to dynamic stability. As MSL has a new backshell shape, it was decided to obtain experimental free flight data of the MSL configuration.

MSL will be the first vehicle since Viking to have a control system actively damping rates during entry, and the vehicle will be the first to maneuver in the Martian atmosphere. The EV will perform a series of bank maneuvers, controlling the direction of the lift vector to manage energy while flying a guided entry towards the desired landing site and attempting to maximize the altitude at which parachute deploy occurs. To properly interpret preflight simulations of the MSL entry, it is important to understand the relative contributions to capsule stability from its inherent dynamic stability and the corrective control authority of the RCS.

Building on Stardust and Genesis experience, the Mars Exploration Rovers mission chose to determine pitch damping characteristics from free-flying models. Viking had used forced oscillation testing to measure pitch damping and Mars Pathfinder (MPF) had used that data for preflight predictions. The MPF entry 
resulted in oscillation amplitude growth significantly exceeding preflight predictions. ${ }^{5}$ It is believed that sting effects can corrupt the mechanisms (wake flow structures) that cause dynamic instabilities in blunt bodies and this resulted in the Viking data underpredicting the MPF dynamic instabilities. ${ }^{6}$ A simulation using MER pitch damping data to reproduce the MPF entry matched much better than did the preflight simulation using Viking forced oscillation data. ${ }^{7}$ Mars Science Laboratory has again returned to the ballistic range to ensure that the geometry changes have not introduced any new dynamic instability issues. Data reduction methods have improved continually as more entry vehicles seek to quantify their dynamic stability using ballistic range techniques.

One area of concern for MSL is the fact that it will be trimming at a non-zero angle-of-attack. While some facilities can test lifting vehicles by operating at low pressures, the pressure reduction required to reduce lift also results in low Reynolds numbers. Free flight data was still judged to be preferable to forced oscillation data. MSL tested non lifting models and is applying extra conservatism to account for lifting effects.

\section{Facility and Methods}

\section{A. Test Facility}

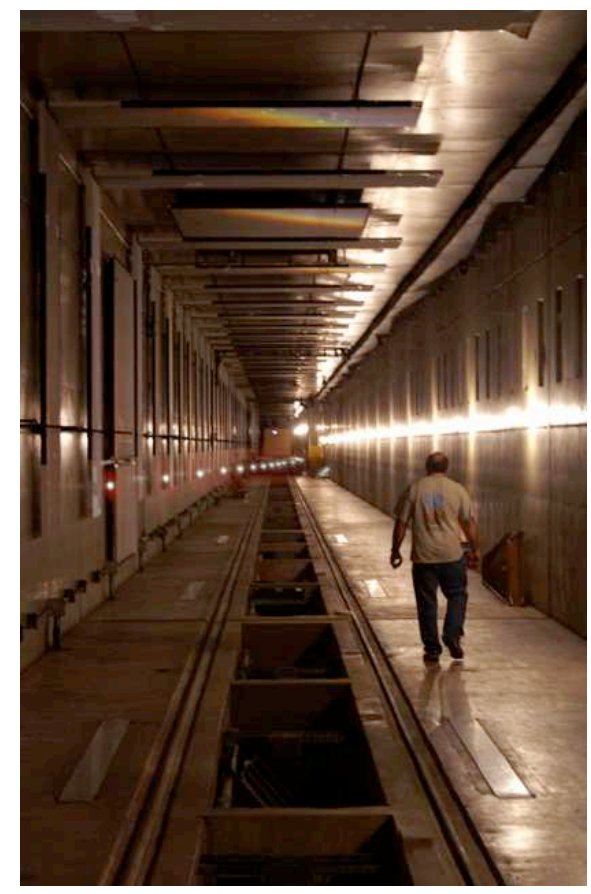

Figure 1. Looking uprange at the Eglin Aeroballistic Research Facility

Freeflight dynamic stability data was collected for the MSL EV at the Aeroballistic Research Facility (ARF) at Eglin Air Force Base, Florida. ${ }^{8}$ This facility is an enclosed concrete structure open to ambient atmosphere, near sea level conditions. The ballistic range consists of a gun room, blast chamber and instrumented range. Models are propelled to test velocity in a three-inch $(76 \mathrm{~mm})$ artillery gun. To launch the MSL EV models from the gun, they are held in four-petal sabots made of polycarbonate plastic. The gun is fired into the blast chamber where all spent propellant and blast products are collected. As the model/sabot package enters the blast chamber, the sabot petals peel off and are arrested by steel plates at the far end of the chamber. The model passes through an aperture into the instrumented range. The instrumented range (see Figure 1) is a $207 \mathrm{~m}$ hallway. The walls are $3.66 \times 3.66 \mathrm{~m}$ square for the first $69 \mathrm{~m}$ of flight, then open to $4.88 \mathrm{~m} \times 4.88 \mathrm{~m}$ for the remaining distance downrange. Fifty sites along the range house orthogonal spark-shadowgraph stations. The fields-of-view of the shadowgraph stations are $2.13 \mathrm{~m}$ diameter circles. As the models fly downrange they break IR switches at each station that initiate spark light sources 
which produce shadows of the model and shock structure on reflective screens opposite the spark sources. Cameras located along one hallway wall and in a pit in the floor of the range take images (shadowgraphs) of each screen. Beads placed on catenary wires provide surveyed reference points in each image to precisely locate the position and orientation of the model at each station. A chronograph is synchronized with the spark source discharge to mark the time each image was captured. The aerodynamic coefficients which best recreate the observed motion of the model are then extracted by parameter identification techniques. ${ }^{9}$

\section{B. Model Geometry}

The ballistic range model tested is a scaled version of a slightly simplified MSL EV geometry. All surface protuberances on the flight EV were considered too small to require modeling for dynamic stability testing. Therefore, the MSL EV model is a body of revolution approximating the overall shape of the vehicle. The models tested had a overall diameter of $65.05 \mathrm{~mm}$ and an overall length of $41.56 \mathrm{~mm}$. This is a $1.44 \%$ scale model of the flight vehicle. To get the axial cg position correct, the model was made in two pieces of different densities. The forebody was made of steel, while the aftbody was made of tungsten and press-fit into the forebody. Figure 2 shows the model geometry and assembly of the two components. Table 1 shows the design mass properties as given by the 3D CAD package used to design the models. The mass properties are based on the solid model and measured densities of the metals used to fabricate the models. Figure 3 shows the ballistic range model and the sabot used for launching the models.

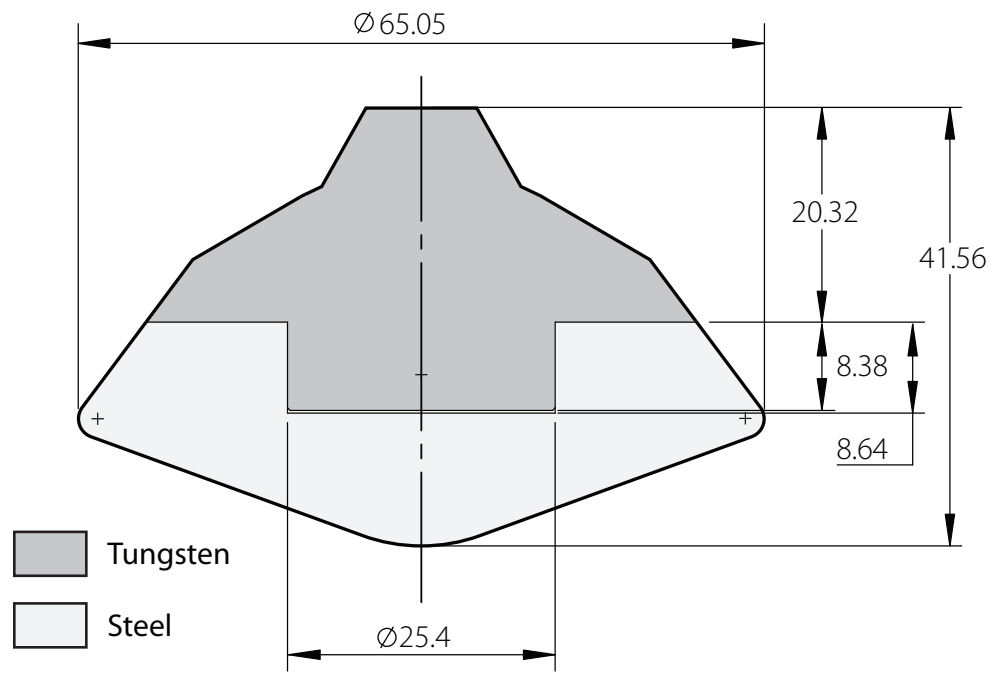

Figure 2. Cross-section of ballistic range model (dimensions in millimeters)

Table 1. Design Mass Properties of Eglin Ballistic Range Model

\begin{tabular}{|c|c|c|c|c|}
\hline $\begin{array}{c}\text { Model Mass Properties } \\
\text { (designed) }\end{array}$ & $\begin{array}{l}\text { Mass } \\
(g)\end{array}$ & $\begin{array}{c}I_{x x} \\
\left(g-m m^{2}\right) \\
\end{array}$ & $\begin{array}{c}I_{y y, z z} \\
\left(g-m m^{2}\right) \\
\end{array}$ & $\frac{x_{c g}}{D}$ \\
\hline & 669 & 2079.2 & 1459.3 & 0.2991 \\
\hline Material Density & $\begin{array}{c}\text { Steel } \\
\left(\mathrm{g} / \mathrm{cm}^{3}\right)\end{array}$ & $\begin{array}{c}\text { Tungsten (Billets 1-3) } \\
\left(\mathrm{g} / \mathrm{mm}^{3}\right) \text {, measured }\end{array}$ & $\begin{array}{l}\text { Tungsten (Billet } 4) \\
\left(\mathrm{g} / \mathrm{mm}^{3}\right), \text { measured }\end{array}$ & \\
\hline & 0.007850 & 0.01689 & 0.01787 & \\
\hline
\end{tabular}

There was no attempt to achieve a radial offset for these models. Pre-test analysis showed that lifting flight would result in swerving trajectories that could not be aimed to fly any significant distance down 
the ARF without striking the hallway. Therefore, all test data is for a non-lifting geometry. Just prior to parachute deploy, the MSL EV jettisons a number of balance masses to move the center of gravity back to an axisymmetric, non-lifting configuration. From Mach 2.5 down to Mach 2.0 the MSL flight cg location is incrementally migrating to match the configuration tested in the ballistic range.

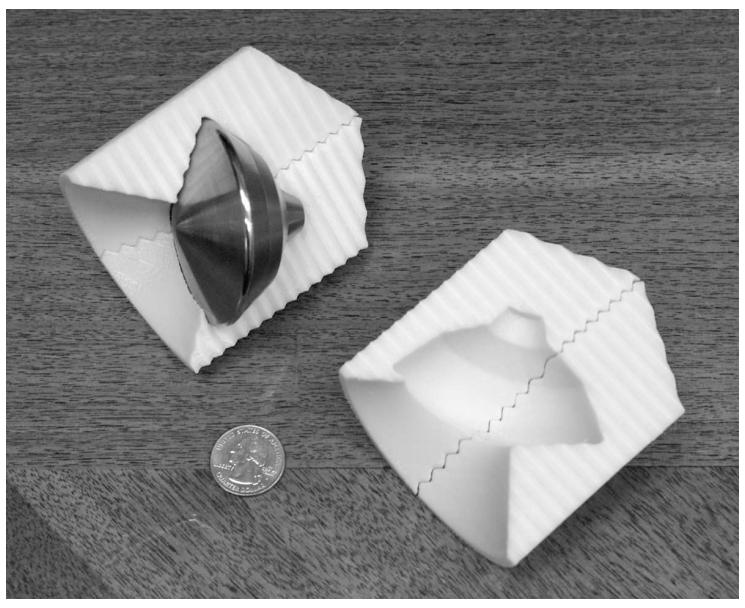

Figure 3. MSL EV ballistic range model and sabot

\section{Test Matrix}

The matrix of test shots was selected to evenly populate an oscillation-amplitude/Mach space where dynamic instabilities have historically been observed and through which the MSL entry vehicle will be flying. The Mach range of interest was from approximately Mach 3.70 down to Mach 1.5. The angle-of-oscillation range varied from $0^{\circ}$ to over $30^{\circ}$. The polycarbonate sabots that held the models in the gun barrel, were fabricated with model cavities canted at different angles relative to the axis of the gun, allowing the models to be held and launched at fixed initial angles-of-attack. This technique provides fairly accurate control over the initial oscillation amplitude. With proper sabot selection and gunpowder mass (selected from historical tables specific to the gun), the test matrix achieved an even data distribution over the desired range of oscillation amplitudes and velocities. As is typical, low speed, low angle data was difficult to obtain. Any small disturbances tend to grow due to dynamic instabilities and decreasing dynamic pressure as the models slow down. The lowest oscillation amplitude data is always obtained at the first data stations. Sabot separation induces some amount of oscillation, so even the first data points for $0^{\circ}$ sabot shots often see amplitudes of $3-5^{\circ}$ or so. The completed test matrix is given in Table 2 .

An example of the shadowgraphs taken at each station are shown in Figure 4. The images were both taken at station 18 during shot S070325 (second of the test matrix) at Mach 2.7. The strong density gradients behind the bowshock cause the forebody shadow to look deformed. Therefore angle measurements are based on the backshell shape. The accuracy with which angles are measured in each shadowgraph is $\pm 0.5^{\circ}$.

\section{Data Reduction Methods}

Two independent data reductions were performed on data collected in the ballistic range. Arrow Tech Associates and AerospaceComputing Incorporated are contractors that have worked closely with the Eglin facility for many years, developing the software for data capture as well as data reduction. Both used their proprietary parameter identification codes to extract static aerodynamics and pitch damping characteristics. AerospaceComputing authored the Comprehensive Automated Data Reduction and Analysis $\left(\mathrm{CADRA}^{10}\right)$ software used by the ARF to measure position and orientation angles from the shadowgraphs of each shot. Therefore, AerospaceComputing assumed the task of extracting raw position/orientation data. This data 
Table 2. MSL EV Ballistic Range Test Matrix, March/April 2007

\begin{tabular}{ccccccc}
\hline Shot No. & Model No. & $\begin{array}{c}\text { Mass } \\
(\mathrm{kg})\end{array}$ & $\begin{array}{c}x_{c g} \\
D\end{array}$ & $\begin{array}{c}I_{y y, z z} \\
\left(\mathrm{~g}-\mathrm{cm}^{2}\right)\end{array}$ & $\begin{array}{c}M_{i} \\
\left(\mathrm{~g}-\mathrm{cm}^{2}\right)\end{array}$ & $\begin{array}{c}\text { Sabot Angle } \\
(\mathrm{deg})\end{array}$ \\
\hline \hline$S 070324$ & 112 & 672.36 & 0.299 & 1474.00 & 3.48 & $10^{\circ}$ \\
$S 070325$ & 121 & 670.14 & 0.299 & 1489.72 & 3.67 & $20^{\circ}$ \\
$S 070326$ & 124 & 668.74 & 0.299 & 1488.31 & 3.59 & $30^{\circ}$ \\
$S 070327$ & 102 & 668.64 & 0.300 & 1470.06 & 3.62 & $20^{\circ}$ \\
$S 070328$ & 109 & 671.03 & 0.299 & 1473.50 & 2.69 & $0^{\circ}$ \\
$S 070329$ & 107 & 663.88 & 0.299 & 1454.84 & 2.59 & $0^{\circ}$ \\
$S 70330$ & 116 & 673.19 & 0.299 & 1477.69 & 2.68 & $10^{\circ}$ \\
$S 070331$ & 101 & 672.32 & 0.299 & 1475.01 & 2.67 & $20^{\circ}$ \\
$S 070332$ & 126 & 667.53 & 0.298 & 1485.83 & 2.62 & $30^{\circ}$ \\
$S 070333$ & 105 & 673.46 & 0.300 & 1476.57 & 3.52 & $0^{\circ}$ \\
$S 070334$ & 110 & 671.10 & 0.299 & 1469.94 & 3.59 & $0^{\circ}$ \\
$S 070335$ & 115 & 671.75 & 0.299 & 1475.97 & 3.51 & $10^{\circ}$ \\
$S 070336$ & 127 & 667.94 & 0.298 & 1490.02 & 3.48 & $30^{\circ}$ \\
$S 070337$ & 114 & 669.35 & 0.299 & 1469.61 & 2.65 & $10^{\circ}$ \\
$S 070338$ & 120 & 669.83 & 0.299 & 1471.38 & 2.59 & $20^{\circ}$ \\
$S 070339$ & 125 & 669.21 & 0.298 & 1489.87 & 2.46 & $30^{\circ}$ \\
$S 070340$ & 108 & 672.76 & 0.299 & 1474.48 & 2.10 & $0^{\circ}$ \\
$S 070341$ & 111 & 671.31 & 0.299 & 1470.78 & 2.14 & $10^{\circ}$ \\
$S 070342$ & 117 & 673.08 & 0.299 & 1476.73 & 2.04 & $20^{\circ}$ \\
$S 070343$ & 103 & 670.88 & 0.299 & 1471.48 & 2.10 & $30^{\circ}$ \\
$S 070344$ & 106 & 671.23 & 0.299 & 1467.64 & 1.49 & $0^{\circ}$ \\
$S 070345$ & 119 & 671.19 & 0.299 & 1471.77 & 1.60 & $0^{\circ}$ \\
$S 070346$ & 118 & 673.16 & 0.300 & 1477.85 & 2.62 & $0^{\circ}$ \\
$S 070347$ & 104 & 668.64 & 0.299 & 1464.20 & 2.63 & $30^{\circ}$ \\
$S 070348$ & $107\left(2^{\text {nd }}\right)$ & 663.84 & 0.299 & 1454.84 & 3.12 & $0^{\circ}$ \\
$S 070349$ & $108\left(2^{n d}\right)$ & 672.77 & 0.299 & 1474.48 & 2.85 & $0^{\circ}$ \\
\hline
\end{tabular}

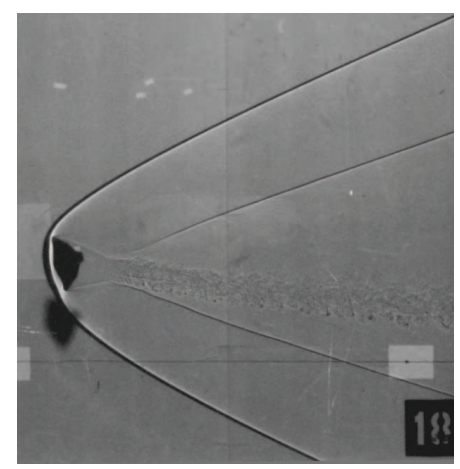

Hall Image

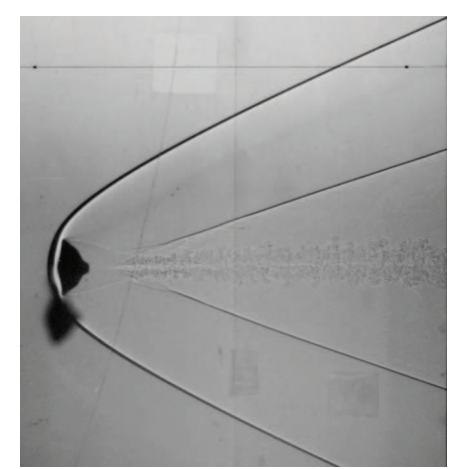

Pit Image

Figure 4. Shadowgraphs of shot S070325 at station 18, Approximate conditions: $\operatorname{Mach}=2.7, \alpha=13^{\circ}, \beta=0^{\circ}$ 
was then used by both AerospaceComputing and Arrow Tech Associates for independent analyses and identification of aerodynamic coefficients.

\section{Arrow Tech Associates ${ }^{11}$}

Arrow Tech uses the Aeroballistic Research Facility Data Analysis System (ARFDAS) ${ }^{12}$ parameter identification code to extract aerodynamic coefficients from ballistic range data. For MSL and other blunt vehicles, ARFDAS first uses 6-degree-of-freedom (DOF) analysis of small sections of ballistic range shots, grouped by angle-of-attack and Mach number, to provide linear pitch damping fits over these small sections. After inputing the ballistic range data including time, position, attitude of the model at each station, mass properties, and ambient atmospheric conditions, ARFDAS performs a sectional analysis to roughly map out the behavior of each coefficient as a function of angle-of-attack and Mach number. The full nonlinear 6-DOF analysis provides the final aerodynamics. From the sectional fits, an estimated table of coefficients is supplied to the 6-DOF program. The 6-DOF analysis uses a Maximum Likelihood Method to iteratively adjust the aerodynamic coefficients, finding the best fit. Adjustments required to capture nonlinear dependencies on angle-of-attack and Mach number are also incorporated in this analysis. ARFDAS also has the ability to perform the 6-DOF analysis on several similar data sets to get the best fit among them.

\section{AerospaceComputing Inc. ${ }^{13}$}

The CADRA program used to scan images is also the AerospaceComputing data reduction tool used to extract aerodynamic coefficients. Similar to the ARFDAS analysis, AerospaceComputing uses linear theory to roughly map out nonlinear pitch damping curves versus Mach number and angle-of-attack. A nonlinear analysis follows to provide the final aerodynamics. AerospaceComputing uses a three step process. First, runs are divided into short segments, where velocity and oscillation amplitude may be considered constant. These small segments help establish aerodynamic trends with Mach number and angle-of-attack. Next, simultaneous linear analysis of the shots with small oscillation amplitudes helps define the nonlinear behavior of the models at small angles-of-attack. Finally, nonlinear analysis is applied to all of the shots. This analysis involves a study to determine the functional form of the pitch damping curve, followed by the determination of nonlinear aerodynamic coefficient curves by fitting 6 -DoF simulations through all the shots simultaneously. Figure 5 shows an example of a CADRA reconstruction, fit through the raw orientation data for one of the shots. This reconstructed trajectory uses the final extracted aerodynamic coefficients described in the next section.

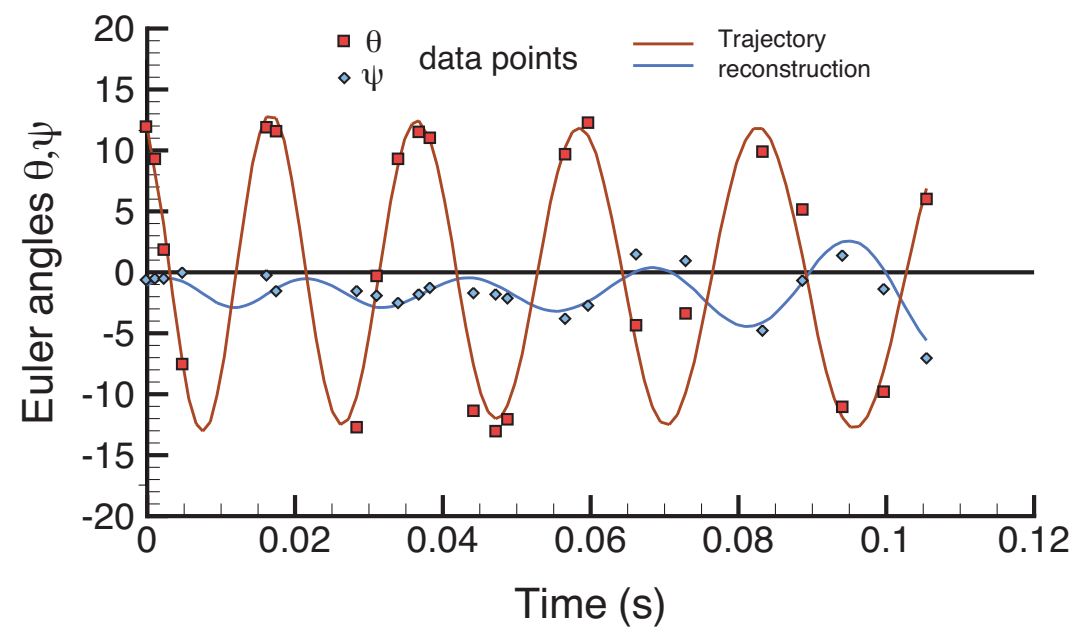

Figure 5. Example of CADRA2 data reduction compared to raw angle measurements for shot S070325. 


\section{Ballistic Range Results}

The 26 ballistic range shots provided a large data set from which pitch damping characteristics of the model were determined. At the higher velocities tested, where Viking, MPF, and MER data suggested there would be dynamically stable behavior, the ballistic range shots did show the capsule to be dynamically stable. Overall, the two data reduction efforts identified similar dynamic stability characteristics. When applied to trajectory simulations, both sets of dynamic aerodynamics produce behavior much like the MER dynamics. The actual curves, which are functions of local angle of attack, appear quite different in form. The curves from both data reduction efforts will be presented, followed by a discussion of these differences.

As predicted, the ballistic range tests show the MSL capsule to be dynamically unstable at small angles starting between Mach 2.8 and 3.25. This instability increases as the model decelerates (data presented goes down to Mach 1). The data extracted by Arrow Tech Associates is very similar to MER ballistic range data and found to be slightly more dynamically stable than the MER capsule. The AerospaceComputing results show some new features not identified in past ballistic range testing. A very rapid change in low-angle dynamic stability was identified as the models decelerated through a specific Mach number. Below this Mach number, a narrow region of dynamic instability with a large peak exists at $\alpha=0^{\circ}$, with positive damping at all angles away from the localized peak. As the MSL EV decelerates further, the peak of the dynamically unstable region decreases, but extends to larger angles of attack. The AerospaceComputing analysts feel that this behavior may have been present in the MER data and its identification here reflects improvement in their data reduction analysis capabilities (augmented/guided by recent forced oscillation test data gathered for the Orion Crew Exploration Vehicle (CEV)). ${ }^{14}$ In contrast, the Arrow Tech analysis was conducted in much the same manner as has been done for past tests, with similar results.

It should be noted that the parameter extracted by both Arrrow Tech and AerospaceComputing is the combined damping coefficient, $C_{m_{q}}+C_{m_{\dot{\alpha}}}$. The separate damping terms due to pitch and angle-of-attack rates are not easy to separate in ballistic range testing and they are typically lumped together. $C_{m_{q}}$ is used here as shorthand for the full coefficient.

\section{A. Static Aerodynamics}

Static aerodynamics extracted by AerospaceComputing and Arrow Tech are in very good agreement with earlier ballistic range test results for the MER entry capsule. ${ }^{7}$ The MER ballistic range data is also in good agreement with CFD-based MER aerodynamic database ${ }^{15}$ and old Viking data. ${ }^{16}$ For brevity, the MSL static force and moment data is not presented. Refer to the earlier MER or Viking data for the static characteristics of $70^{\circ}$ sphere-cone blunt bodies.

\section{B. Arrow Tech Associates Damping Results}

The expression for the pitch damping coefficient used in the 6-DOF analysis is:

$$
C_{m_{q}}=C_{m_{q, \text { initial }}}+C_{m_{q, 2}} \varepsilon^{2}+C_{m_{q, 4}} \varepsilon^{4}+C_{m_{q, m}}\left(M_{i}-M_{r e f}\right)+C_{m_{q, m 2}}\left(M_{i}-M_{r e f}\right)^{2}
$$

where

$$
\varepsilon=\sin \left(\alpha_{T}\right)=\sqrt{\frac{V_{z}^{2}+V_{y}^{2}}{V^{2}}}
$$

and

$$
M_{r e f}=2.5
$$

$M_{r e f}$ is an arbitrary constant used in the data reduction. A table of $C_{m_{q, \text { initial }}}$ coefficients are determined from linear fits using sections of individual shots. This table is an initial estimate of the nonlinear pitch damping coefficients. The table of initial coefficients is then corrected to better resolve Mach number and angle-of-attack variations by fitting 6 -DoF trajectories through the data points of multiple, complete ballistic range shots. The final table of coefficients, listed in Table 3, includes the extra terms in Equation 1 and reflects the identified dependencies on angle of attack and Mach number. 
Table 3. Arrow Tech Associates supersonic $C_{m_{q}}$ results, $x_{c g} / d=0.30$

\begin{tabular}{llllllllll}
\multicolumn{10}{c}{ Mach } \\
$\alpha$ & 1.35 & 1.5 & 1.75 & 2.0 & 2.25 & 2.5 & \multicolumn{1}{l}{3.0} & \multicolumn{1}{l}{3.5} & \multicolumn{1}{l}{4.0} \\
0 & 0.9878 & 0.9093 & 0.7246 & 0.5652 & 0.4442 & 0.3231 & 0.1098 & -0.0900 & -0.2898 \\
2 & 0.8882 & 0.8127 & 0.6402 & 0.4914 & 0.3753 & 0.2592 & 0.0441 & -0.1422 & -0.3285 \\
4 & 0.6438 & 0.5765 & 0.4321 & 0.3078 & 0.2052 & 0.1026 & -0.1107 & -0.2655 & -0.4212 \\
6 & 0.3650 & 0.3066 & 0.1950 & 0.0990 & 0.0113 & -0.0765 & -0.2700 & -0.3933 & -0.5175 \\
8 & 0.1457 & 0.0945 & 0.0087 & -0.0648 & -0.1409 & -0.2169 & -0.3789 & -0.4806 & -0.5823 \\
10 & 0.0153 & -0.0315 & -0.1023 & -0.1629 & -0.2318 & -0.3006 & -0.4329 & -0.5238 & -0.6147 \\
12 & -0.0456 & -0.0903 & -0.1542 & -0.2088 & -0.2745 & -0.3402 & -0.4536 & -0.5400 & -0.6273 \\
14 & -0.0693 & -0.1134 & -0.1741 & -0.2259 & -0.2903 & -0.3546 & -0.4590 & -0.5445 & -0.6309 \\
16 & -0.0757 & -0.1197 & -0.1799 & -0.2313 & -0.2952 & -0.3591 & -0.4599 & -0.5454 & -0.6309 \\
18 & -0.0771 & -0.1208 & -0.1809 & -0.2322 & -0.2961 & -0.3600 & -0.4608 & -0.5463 & -0.6318 \\
20 & -0.0779 & -0.1218 & -0.1814 & -0.2322 & -0.2966 & -0.3609 & -0.4608 & -0.5463 & -0.6318 \\
25 & -0.0779 & -0.1218 & -0.1814 & -0.2322 & -0.2966 & -0.3609 & -0.4608 & -0.5463 & -0.6318 \\
30 & -0.0779 & -0.1218 & -0.1814 & -0.2322 & -0.2966 & -0.3609 & -0.4608 & -0.5463 & -0.6318
\end{tabular}

The pitch damping coefficient, $C_{m_{q}}$, is plotted in Figure 6 for a range of Mach numbers from Mach 3.5 down to Mach 1.4. These curves, interpolated from the final coefficient matrix in Table 3, show the capsule to be dynamically unstable $\left(C_{m_{q}}>0\right)$ at low angles-of-attack. The magnitude of instibility is seen to increase as the MER capsule decelerates towards Mach 1.4. At higher angles-of-attack, the capsule is seen to be stable but becomes less so with decreasing Mach number. The Mach variation is roughly a linear shift of the entire pitch damping curve. Figure 7 shows plots of total angle-of-attack and Mach number versus downrange position for three representative shots. The initial angles-of-attack were similar for each shot, so the difference in amplitude growth between them is due to the dynamic stability variation with Mach number. The slower the initial velocity, the greater the amplitude growth downrange. The pitch damping curves and behaviors observed are generally similar to the Arrow Tech Associates MER data.

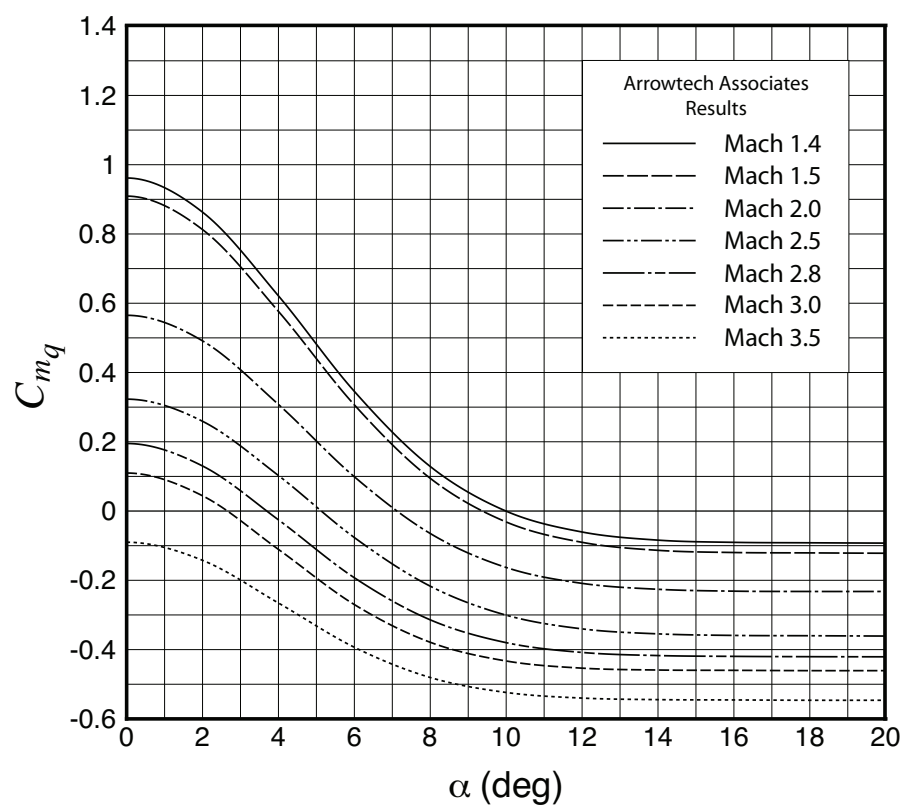

Figure 6. Arrow Tech Associates supersonic pitch damping coefficient curves, $C_{m_{q}}\left(x_{c g} / d=0.30, z_{c g} / d=0.0\right)$ 


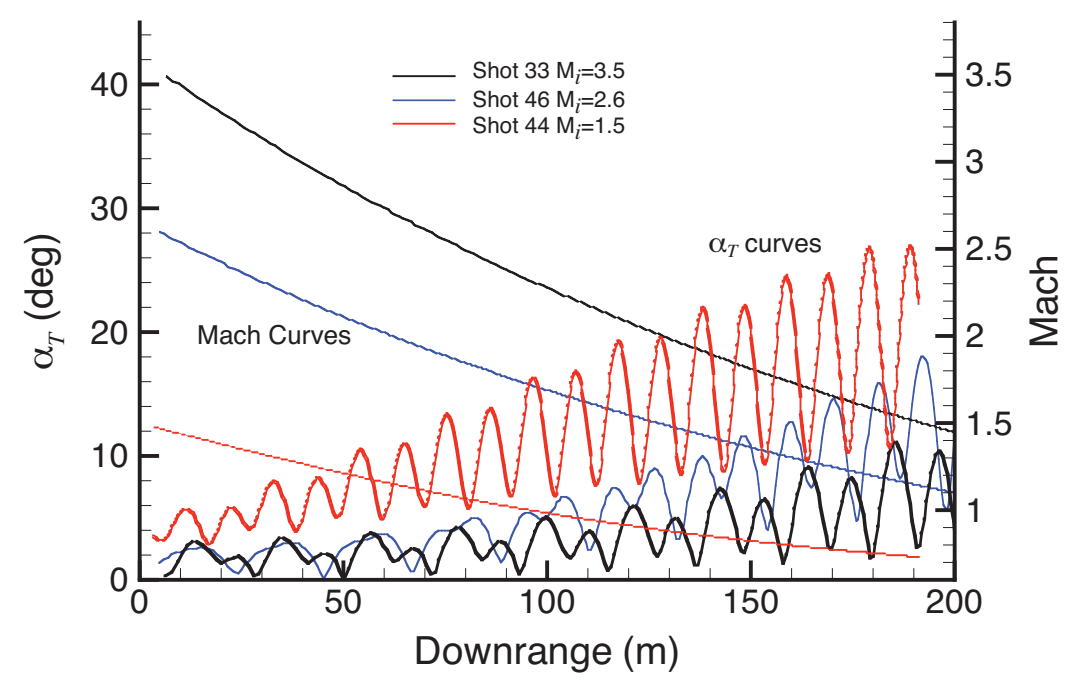

Figure 7. Example of amplitude growth with initial Mach number

\section{AerospaceComputing Results}

The AerospaceComputing pitch damping coefficient data is plotted in Figure 8 for a range of Mach numbers versus angle-of-attack. The functional form describing these curves is:

$$
\begin{gathered}
M_{\infty}<2.8, \quad C_{m_{q}}=\max \left[\left(C_{m_{q, 1}}+C_{m_{q, 2}} M_{\infty}+C_{m_{q, 3}} M_{\infty}^{2}+C_{m_{q, 4}} M_{\infty}^{2} \sin ^{2} \alpha\right),\left(C_{m_{q, 5}}+\frac{C_{m_{q, 6}}}{M_{\infty}^{2}}\right)\right] \\
M_{\infty}>2.8, \quad C_{m_{q}}=C_{m_{q, 5}}+\frac{C_{m_{q, 6}}}{M_{\infty}^{2}}
\end{gathered}
$$

Coefficients for these functions were determined by multiple fits of the ballistic range shots with 6 -DOF nonlinear analysis. These coefficients are

$$
\begin{gathered}
C_{m_{q, 1}}=1.5733 \\
C_{m_{q, 2}}=-0.9797 \\
C_{m_{q, 3}}=0.4985 \\
C_{m_{q, 4}}=-30.9 \\
C_{m_{q, 5}}=-0.47 \\
C_{m_{q, 6}}=0.16
\end{gathered}
$$

The curves show interesting behavior with angle of attack and Mach number. The AerospaceComputing results indicate that there is a distinct Mach number as the MSL EV decelerates, M $=2.8$, where a dynamic instability arises. The peak of this instability at $\alpha=0^{\circ}$ is very large, but only extends about two degrees from the peak. At lower Mach numbers, this peak decreases, but extends to larger angles-of-attack. This instantaneous onset of dynamic stability with a very narrow, but steep peak has not been identified in other ballistic range data analysis (MER, Genesis, Stardust, etc.). However, features like this have recently been identified in forced oscillation testing of the CEV (Orion) command module. This is thought to be reflective of the advancements in data reduction technique and the increasing knowledge base of the data analysts, rather than a distinct behavior exclusive to the MSL entry vehicle. While the functional form differs greatly from the Arrow Tech Associates results, the integrated effects of both sets of data, when used in simulation, replicate the measured data sets very well. A comparison of the integrated effects is presented next. 


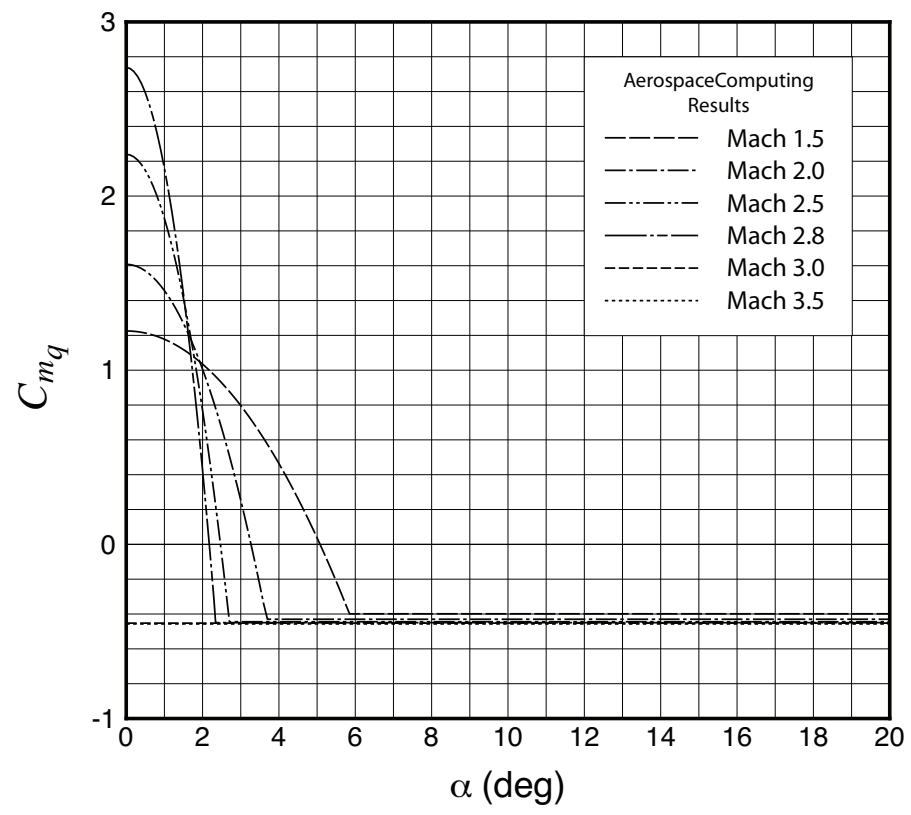

Figure 8. AerospaceComputing supersonic pitch damping coefficient curves, $C_{m_{q}}\left(x_{c g} / d=0.30, z_{c g} / d=0.0\right)$

\section{Discussion of Arrow Tech and AerospaceComputing Results}

The functional forms of AerospaceComputing and Arrow Tech Associates results are quite different. While the Arrow Tech results are very similar to MER results, the form of the AerospaceComputing damping curves are very different than the measured dynamic stability curves of past Mars and Earth return missions. To compare them, the mean value theorem is used to determine the integrated effect of dynamic damping for a given oscillation amplitude. This integrated effect allows the user to replace a very nonlinear curve with a constant value. At a given oscillation amplitude, the constant value will produce oscillation growth or decay identical to the nonlinear curve. This provides a useful way to compare two very different nonlinear damping curves and assess how they will drive oscillatory behavior in flight. The application of the mean value theorem to express damping as function of oscillation amplitude was well documented by Redd et al. ${ }^{17}$ The exact application used here is described by Schoenenberger and Queen. ${ }^{18}$ The effective dynamic damping for a decelerating blunt body in a constant density flowfield is given by

$$
\overline{C_{m_{q}}}=\frac{\int_{0}^{\frac{2 \pi}{\omega}}\left(C_{m_{q}}+C_{m_{\dot{\alpha}}}\right) \dot{\alpha}^{2} d t}{\int_{0}^{\frac{2 \pi}{\omega}} \dot{\alpha}^{2} d t}
$$

Figure 9 shows the effective damping versus oscillation amplitude for the AerospaceComputing and Arrow Tech results. At Mach=2.8 and lower, the AerospaceComputing results show the dynamic stability to be much more unstable at small angles of attack. However, the AerospaceComputing curves collapse to behave much like the Arrow Tech data when oscillating at amplitudes of $10^{\circ}$ or greater, and the effective damping is almost independent of Mach number. The low angle-of-attack damping trends toward becoming dynamically stable as the capsule decelerates, following the discrete jump at Mach=2.8. When used in simulation, the AerospaceComputing and Arrow Tech curves will add energy over approximately the same small angle range and remove energy over the same large angle range. The large positive $C_{m_{q}}$ values seen at the small angles of the AerospaceComputing curves will cause the capsule to grow to neutral energy oscillation amplitudes much faster than the Arrow Tech curves. This is the chief difference between results from these two data reduction efforts. The results are in closest agreement at moderate oscillation amplitudes $\left(\alpha=10^{\circ}\right.$ to $\left.20^{\circ}\right)$ near Mach 2.5. The majority of ballistic range shots passed through this oscillation-amplitude and Mach space and the curves are therefore anchored by the most number of data points in this space.

The oscillation amplitudes where $\overline{C_{m_{q}}}$ crosses zero is the point where the dynamic damping does not add 


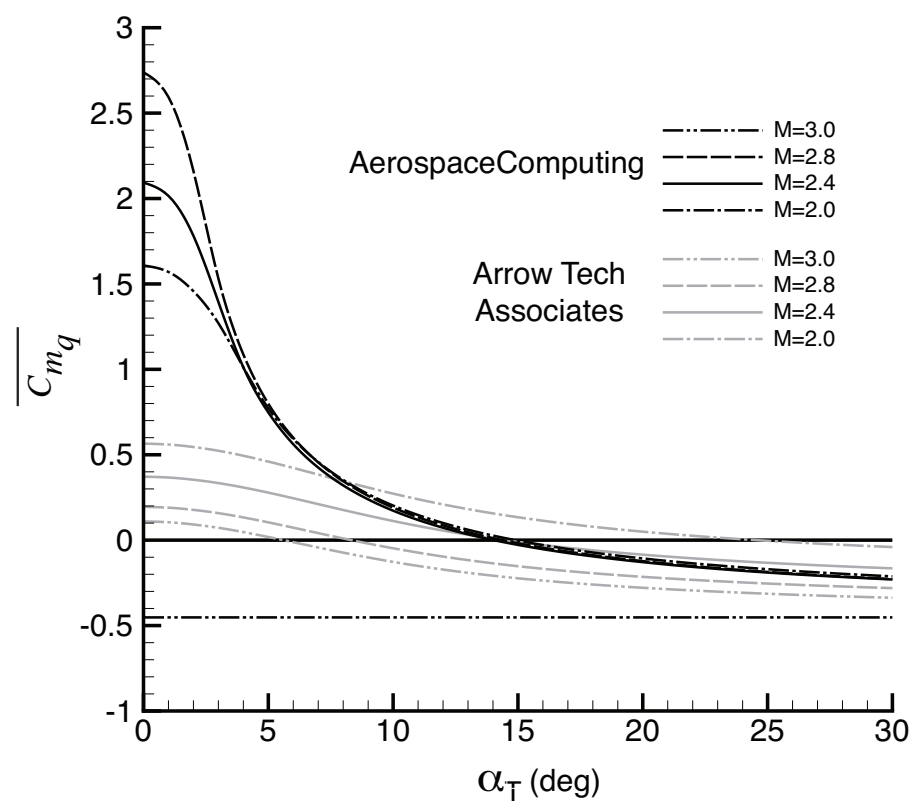

Figure 9. Effective damping for several Mach numbers, $\left(x_{c g} / d=0.30, z_{c g} / d=0.0\right)$

to or subtract from the oscillatory energy of a body in free flight. A plot of these constant energy amplitudes for both sets of data is shown in Figure 10. Note the crossing point where the two sets of data agree is at Mach 2.4 and an oscillation amplitude of about $15^{\circ}$. The discrete jump of the AerospaceComputing data at Mach $=2.8$ is also obvious. Above that point, the AerospaceComputing data indicate that capsule oscillations are losing energy at all amplitudes. The Arrow Tech data indicate a similar falloff in constantenergy amplitude with increasing Mach number. The capsule must be at speeds above Mach 3.27 before the dynamic term is stable for all angles-of-attack. At low speeds, the Arrow Tech data indicates the capsule must be oscillating about amplitudes much greater than those of the AerospaceComputing data to achieve a constant energy oscillation. However, as shown in Figure 8, the AerospaceComputing damping model causes much faster amplitude growth at low angles, up to the constant energy amplitude. In the ballistic range, the Mach number is changing very rapidly and the flight time is so short that oscillation amplitude growth or decay generally never reaches equilibrium during a shot. The determination of the exact functional form with this test technique may not be possible. In general, where there is the most data to anchor the curves, these two analysis efforts are in closest agreement. For use in flight simulation it is conservative to use the curves which produce the most destabilizing behavior. Since the MSL vehicle is controlled, it will be oscillating about small amplitudes. Therefore, the AerospaceComputing data which causes rapid growth up to moderate amplitudes is the more conservative set of data. Above Mach 2.8, it is more conservative to use the Arrow Tech data which still has regions of dynamic instability.

The MER aerodatabase constant energy curve was also calculated and included in Figure 10. As was shown in the Figure 6, the MER and MSL Arrow Tech data is very similar in form, differing roughly by a constant value. The MER data reaches a constant energy point at larger angles of attack and becomes fully stable at Mach 3.5. To be conservative, the MER data will be used in the MSL aerodynamic database at Mach numbers greater than 2.8 .

The constant energy amplitude point is where dynamic stability adds no energy to the system. However, as the MSL capsule is decelerating, oscillation amplitude still increases as the drop in dynamic pressure reduces the restoring static moments acting on the vehicle. The oscillation amplitude growth for a decelerating body in a constant density flowfield can modeled by the Euler-Cauchy equation as described in two papers by Schoenenberger and Queen. ${ }^{18,19}$ The solution of the E-C equation is of the form:

$$
\alpha=A t^{\mu} \cos (\nu \ln t+\delta)
$$




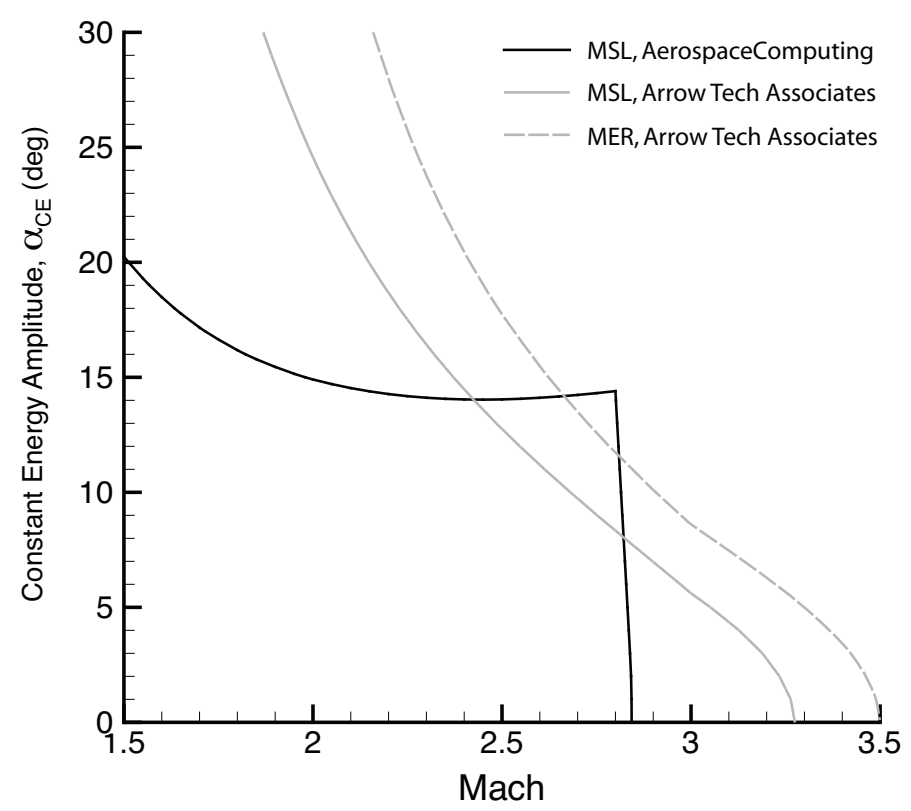

Figure 10. Constant energy amplitude versus Mach number. $\left(x_{c g} / d=0.30, z_{c g} / d=0.0\right)$

Where $\delta$ is phase shift angle, $\nu$ is a frequency term, and $\mu$ is a damping term. The oscillation amplitude of a decelerating capsule grows proportional to time raised to the damping term, $\mu$. This term is:

$$
\mu=\frac{1}{2}-\frac{C_{L_{\alpha}}}{2 C_{A}}+\frac{m d^{2}}{4 I C_{A}} \overline{C_{m_{q}}}
$$

While, $\overline{C_{m_{q}}}=0$ results in constant oscillatory energy, it does not result in constant amplitude oscillations. To achieve constant oscillation amplitude, the dynamic damping must be sufficient to negate the amplitude growth due to deceleration and lift generated as the capsule oscillates. Setting $\mu$ equal to zero the $\overline{C_{m_{q}}}$ value required for constant oscillations is found to be

$$
\bar{C}_{m_{q}, \alpha=\text { constant }}=\left(C_{L_{\alpha}}-C_{A}\right) \frac{2 I}{m d^{2}}
$$

The constant amplitude damping is equal to about -0.27 for the MSL entry vehicle.

When the effective damping satisfies $\mu=0$, the capsule will see no oscillation amplitude growth. Equation 9 shows that the effective damping must be negative to counteract the constant $1 / 2$ term which produces natural oscillation growth due to decreasing dynamic pressure as the EV decelerates, and the $C_{L_{\alpha}}$ term which causes growth from a coupling between heaving due to lift and the oscillations of the capsule. As the body oscillates, the lift force (itself dominated by the pointing of the axial force vector) produces heaving motion that adds an induced component to the angle-of-attack rate. Through this induced rate from the heaving motion, the static aerodynamics alone can affect amplitude growth.

\section{E. Comparison to the Mars Exploration Rover Entry Capsule}

It is useful to compare the MSL ballistic range data to the MER ballistic range data used in the MER flight aerodatabase. As MER and MSL have essentially identical forebody shapes, this comparison shows the impact of changing backshell geometry on the dynamic stability of the vehicle. Figure 11 shows the effective damping of the two capsules as identified by Arrow Tech Associates. Arrow Tech's MER ballistic range results were used in the MER aerodynamic database. The damping functional forms are essentially identical, but the MSL capsule is slightly more stable than MER. The two sets of curves differ by a constant offset roughly equal to a change in Mach number of about 0.2 to 0.3. 
The functional forms were not specifically selected to be of identical form. The similarity of the curves is likely a result of commonality in methods and personnel used by Arrow Tech Associates to extract the dynamic damping coefficients. It is not likely than any physical phenomena can be inferred from the similar results, but this consistency in data reduction analysis adds confidence that the overall MSL dynamic stability characteristics are similar to MER and slightly more stable.

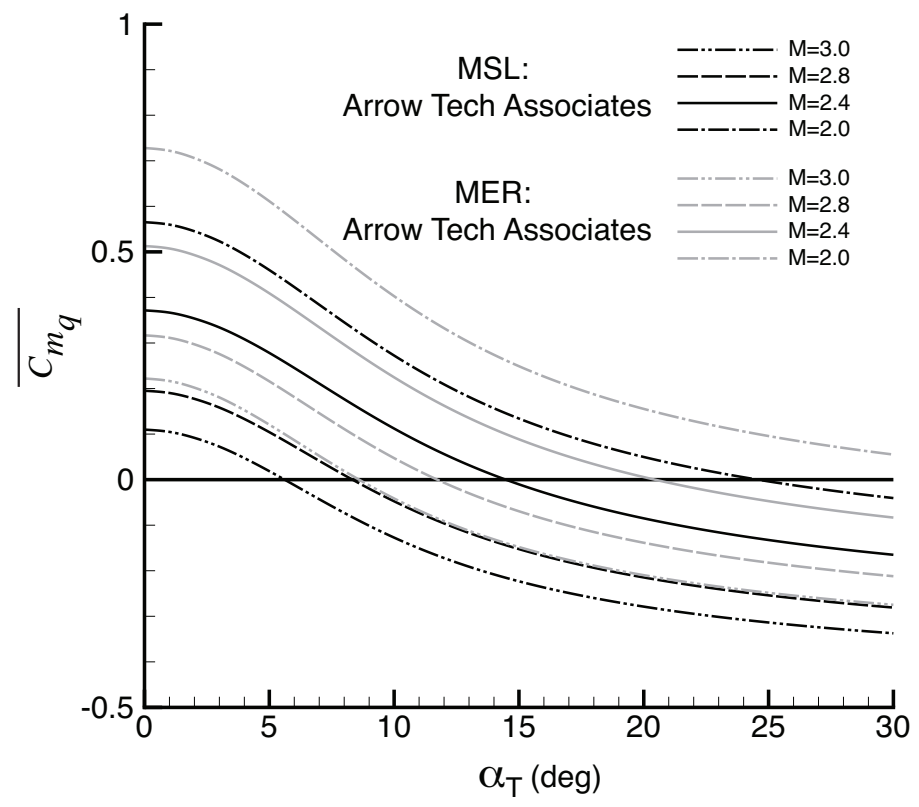

Figure 11. Comparison of MER and MSL effective damping for several Mach numbers, $\left(x_{c g} / d=0.30, z_{c g} / d=0.0\right)$

The functional form identified by AerospaceComputing for the MER ballistic range data was similar to the MER Arrow Tech results. The new functional form identified for the MSL shape is believed to reflect improvements in dynamic stability modeling overall (guided by recent improvements in blunt-body forced oscillation tests) and not a distinct change in the dynamic stability characteristics of the vehicle. A comparison using MER and MSL pitch damping models to replicate the MSL data points was performed to assess their differences. These comparisons showed the integrated effect of the pitch damping curves. While local discrepancies, especially at low oscillation amplitudes (where there are the fewest data points anchoring the models) were observed, in general both models fit the MSL data well. The comparison suggests only small differences in the overall dynamic stability characteristics of the MER and MSL vehicles. The differences in the pitch damping functional forms were not driven by any significant changes to dynamic stability because of backshell geometry changes.

\section{F. Uncertainties}

The uncertainties used for the MSL database are based on those used for MER, but their implementation has been modified to produce more conservative dispersions across the Mach and angle-of-attack space. The pitch and yaw damping dispersion model in the supersonic regime is as follows:

$$
\begin{gathered}
C_{m_{q, \text { disperse }}}=\left(1+U_{q}^{M}\right)\left(C_{m_{q, \text { nominal }}}+0.5\right)-0.5+U_{q}^{A} \\
C_{n_{r, \text { disperse }}}=C_{m_{q, \text { disperse }}}
\end{gathered}
$$

Where the multiplier and adder dispersion values are:

$$
U_{q}^{M}=[-50 \% \text { to }+150 \%]=[-0.5,+1.5]
$$




$$
U_{q}^{A}=[0.00 \text { to }+0.10]
$$

The 0.5 terms in the dispersion model are used to keep negative regions of the pitch damping curves from becoming more negative when a positive dispersion is applied. The curves are shifted up so the pitch damping is zero or positive, the multiplier is applied, and the curves are shifted back down by the same increment. The adder is then applied to disperse the asymptote. The adder value of 0.10 was used in the MER database as a dispersion on the Mach variation of the pitch damping curve. A value of 0.1 corresponded to a change in Mach number of approximately 0.5. The multiplier was selected to disperse the peak dynamic instability. As shown in the two MSL data reduction efforts, the pitch damping at small angles-of-attack is the region with the greatest uncertainty. The multiplier was chosen to be rather large to account for this uncertainty. To be conservative, the multiplier is also biased in the destabilizing direction. The multiplier is dispersed using a Gaussian distribution, while the adder is distributed uniformly.

Another issue regarding uncertainties is that the MSL capsule is trimming at a non-zero angle-of-attack over much of the Mach range covered by this test. At Mach 2.5 the capsule is trimming near $-20^{\circ}$, at which time six entry balance masses $(\sim 29 \mathrm{~kg}$ each) are jettisoned in sequence, smoothly changing the trim angle to $0^{\circ}$ just before parachute deploy at Mach 2.0. If the unaltered pitch damping model were used in simulation, the capsule would experience only positive damping at the large trim angles as the capsule would not see the peak instabilities at $\alpha=0^{\circ}$. Testing with the correct $\mathrm{cg}$ offset would be required to quantify how the damping characteristics change with trim angle. For MSL simulations, the non-lifting data is implemented in the most conservative manner possible; the peak dynamic instability is shifted to the current trim angle-of-attack (accomplished by shifting the angle-of-attack variable used to call the damping model). This modification conservatively accounts for possible radial $\mathrm{cg}$ offset effects and the accompanying non-zero trim angle.

\section{G. Comparison to Reaction Control System (RCS) Authority}

To better understand the magnitude of the MSL dynamic instabilities in the context of controlled flight, the dimensional torques caused by the dynamic damping coefficients are compared to the control authority of the reaction control system. The MSL controller uses four pairs of reaction control jets to perform maneuvers and damp any unwanted rates. Figure 12 shows the reaction control system on the MSL backshell. The jets are scarfed with the nozzle exits approximately flush with the backshell. The jet pairs are canted to provide components of roll, pitch and yaw. The controller selects the proper combination to generate torques about a desired axis. Each jet generates approximately $290 N(65 \mathrm{lb})$. The reaction control system is capable of generating roll and yaw torques of \pm 900 (jets $3,4,7$ and 8 , or 1,2,5 and 6) and $\pm 600 \mathrm{Nm}$ (jets 5,6,7 and 8) respectively. The RCS pitch authority is +1200 (jets 3,4,5 and 6) and $-1000 \mathrm{Nm}$ (jets 1,2,7 and 8). The asymmetry is due to the radial cg offset in the pitch plane.

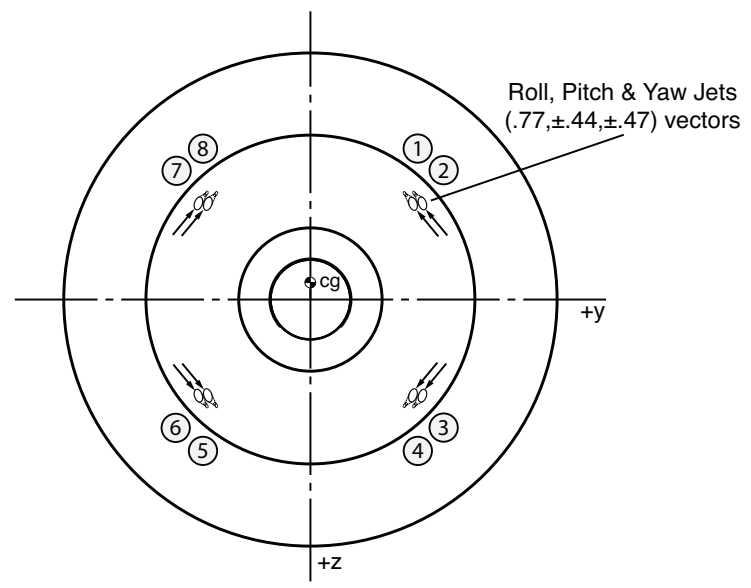

Figure 12. MSL RCS jet configuration, jets are labeled with MSL numbering convention 
To assess the dynamic torques, the damping coefficients are combined with the rates associated with a range of oscillation amplitudes for several points along a nominal reference trajectory. At a given Mach number and dynamic pressure, the static stability can be used to calculate the peak rotational rate the EV will see as it oscillates at the specified amplitude. A sinusoidal oscillation is assumed. The effective peak constant torques and the peak instantaneous torques are plotted here against the nominal MSL RCS pitch torques.

The peak torque is the product of the peak pitch damping value $\left(C_{m_{q}}\right.$ at $\left.\alpha_{\text {Trim }}\right)$ and the peak rate (over an oscillation) which also occurs at the trim angle-of-attack. This the largest instantaneous dynamic torque that the capsule will see for a given oscillation amplitude.

$$
T_{\text {peak }}=C_{m_{q}}\left(\alpha=0^{\circ}\right) \cdot \dot{\alpha}_{\text {peak }}\left(\alpha_{o}\right)
$$

It is useful to compare the peak destabilizing torques to the RCS torques, but note that away from the peak rate are regions where the pitch damping curve is less positive and at large angles, negative. When the EV is at angles away from the angle of peak pitch damping, the controller will be fighting much smaller torques, or helped by regions of positive damping. As shown in Equation 7, the damping can also be expressed as a constant value for a given oscillation amplitude. The effective damping is a constant value that can replace a nonlinear pitch damping curve for a given oscillation amplitude. The effective peak torque is simply the effective damping for a given oscillation amplitude, multiplied by the peak rate for that amplitude. Comparing effective torques against the controller authority shows the capacity of the controller to negate any undamping produced by dynamic instabilities.

$$
T_{\text {effective }}=\overline{C_{m_{q}}}\left(\alpha_{o}\right) \cdot \dot{\alpha}_{\text {peak }}\left(\alpha_{o}\right)
$$

The peak rate for a given oscillation amplitude can be estimated by modeling the EV pitching oscillations as a simple harmonic oscillator:

$$
\ddot{\alpha}-\frac{\rho V_{\infty} S d^{2}}{4 I_{y y}}\left(C_{m_{q}}+C_{m_{\dot{\alpha}}}\right) \dot{\alpha}-\frac{\rho V_{\infty}^{2} S d}{2 I_{y y}} C_{m_{\alpha}} \alpha=0
$$

For constant coefficients, the solution to Equation 17 is:

$$
\alpha=\alpha_{o} e^{\xi t} \sin (\omega t+\delta)
$$

where $\alpha_{o}$ is the oscillation amplitude and the frequency of oscillation is

$$
\omega=\sqrt{-\frac{\rho V_{\infty}^{2} S d}{2 I_{y y}} C_{m_{\alpha}}}
$$

Compared to static moments, the damping moments are small for a single oscillation cycle and the amplitude can be approximated as constant. The peak rate is then

$$
\dot{\alpha}_{\text {peak }}=\alpha_{o} \omega \cos ((\omega t+\delta)=0)=\alpha_{o} \omega=\alpha_{o} \rho^{\frac{1}{2}} V_{\infty}\left(-C_{m_{\alpha}}\right)^{\frac{1}{2}}\left(\frac{S d}{2 I_{y y}}\right)^{\frac{1}{2}}
$$

The peak rate is directly proportional to the oscillation amplitude, the square root of the dynamic pressure and the square root of the static stability. Here the dynamic pressure is broken out to highlight the dependency of the peak rate due to density and velocity as well.

To demonstrate the dimensional torques that dynamic stability terms produce in flight, a typical reference trajectory and the allocated mass properties are used to predict the peak rates for a range of oscillation amplitudes. The local peak torques and effective torques integrated over a full oscillation are then presented and compared to the RCS pitch authority of the MSL EV. The allocated mass and pitching moment of inertia of the MSL EV are

$$
\begin{gathered}
m=3067.0 \mathrm{~kg} \\
I_{y y}=3352.62 \mathrm{~kg}-\mathrm{m}^{2}
\end{gathered}
$$


Figure 13 shows the peak torques that the MSL EV will see at key points along a reference trajectory. These torques are calculated using the $\alpha=0^{\circ} C_{m_{q}}$ data in the MSL database (AerospaceComputing ballistic range data for the Mach 2.0 and 2.8 curves) multiplied by the peak rate that the EV will see for a given oscillation amplitude, given by Equation 19. Note that the hypersonic pitch damping value is negative, so any rates are naturally damped at the point of peak dynamic pressure and any point above Mach 5.0. Mach 2.8 is the point of peak dynamic instability in the aerodatabase and therefore produces the greatest peak adverse torque for a given rate. The $+3 \sigma$ dispersed curve does exceed the RCS control authority, but the EV must be oscillating $\pm 25^{\circ}$ or greater about the trim point for this to occur. Recall that the pitch damping curve is very nonlinear with angle-of-attack at the supersonic Mach numbers, and even this large peak adverse torque will be mitigated significantly as the EV swings through angles-of-attack with positive damping. At Mach 2.0, the peak adverse torque is slightly smaller than at Mach 2.8, and the dynamic pressure has also decreased. This results in a significantly reduced peak torque at parachute deploy conditions. Note each curve in Figure 13 is calculated up to an oscillation amplitude of $30^{\circ}$. The $\alpha_{o}=30^{\circ}$ peak rates at each flight condition (the rate at the end of each line in Figure 13b) give an idea of how the combined effects of the dynamic pressure and static stability vary along the trajectory, changing the influence of the pitch damping data.

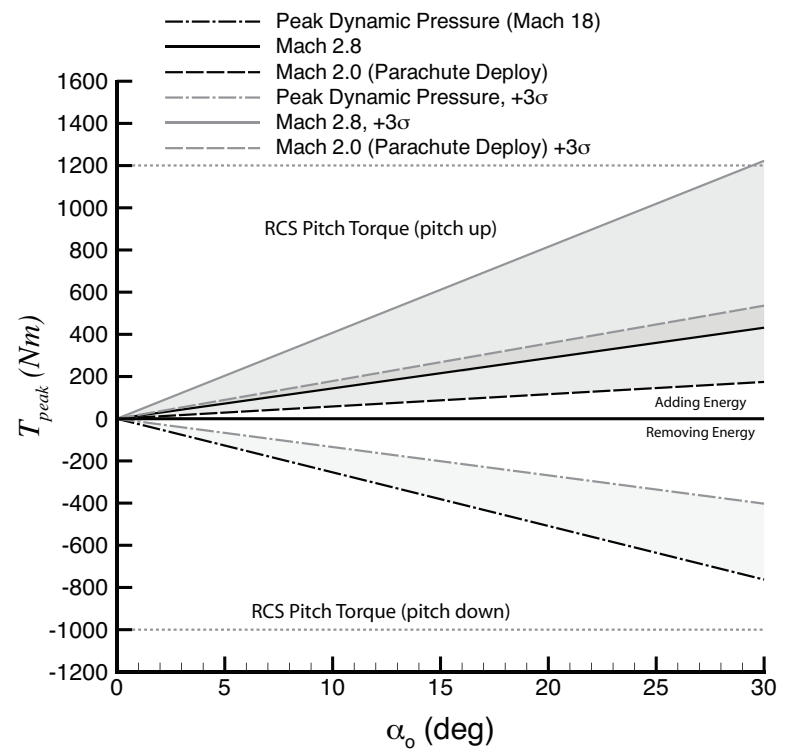

(a) Peak damping vs. amplitude

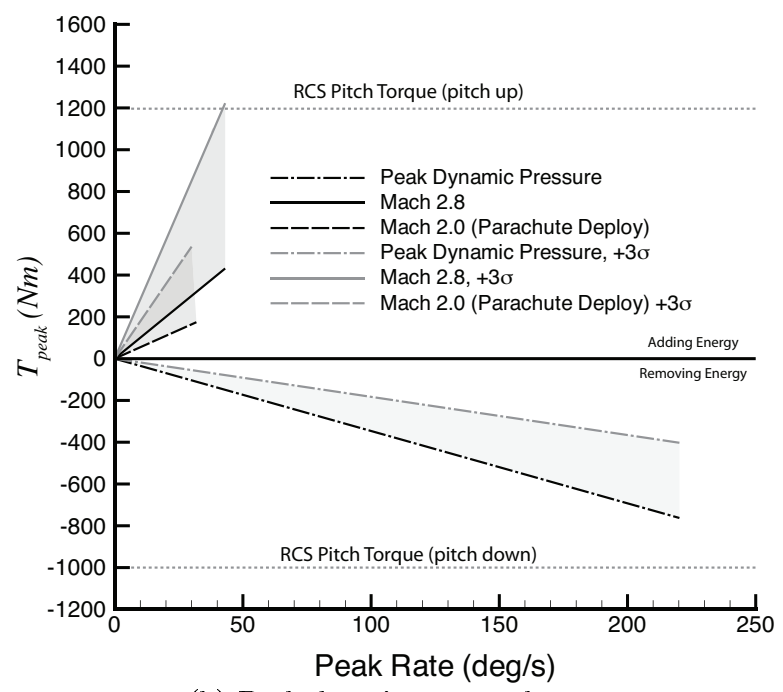

(b) Peak damping vs. peak rate

Figure 13. Peak damping torques (at $\alpha=\alpha_{\text {Trim }}$ ) based on AerospaceComputing BR data at several points along reference trajectory

While it is important to note the peak possible torques that the dynamic stability characteristics can impart on the vehicle, the integrated effect is a more representative indicator of the undamping effects a controller will have to overcome in practice. The mean value theorem (Equation 7) shows that the nonlinear pitch damping curve may be replaced by a constant value for a given oscillation amplitude. Likewise, the product of the damping coefficient and instantaneous angular rate can be integrated over time to express the dynamic torques as a constant value for a given oscillation amplitude. The effective damping torques can be thought of as a direct increase or reduction of the RCS control torques, directly proportional to the instantaneous rate. These damping effects vary with oscillation amplitude (which drives the peak rate and effective damping) and Mach number (which also changes the aerodynamic damping). Figure 14 shows effective torques versus oscillation amplitude and the peak rates that occur for those amplitudes. Again, in Figure 14b the curves were generated for oscillation amplitudes up to $30^{\circ}$. The variation in peak torques for the different curves is due to changes in dynamic stability and the variation in peak rates for the different curves is due to changes in static stability and dynamic pressure along the entry trajectory.

Note that the $+3 \sigma$ peak adverse torque at Mach 2.8 reaches a peak of approximately $75 \mathrm{Nm}$ at $\alpha_{o}=4^{\circ}$. 
For small angle oscillations, the region of dynamic instability dominates the effective damping. The effective damping torques grow nearly linearly due to the proportional increase in rates as amplitude increases. For larger oscillation amplitudes, the negative $C_{m_{q}}$ values at larger angles of attack provide more positive damping, resulting in a gradually decreasing effective damping for any reasonable oscillation the MSL EV might see. At hypersonic speeds (represented here by the peak dynamic pressure curves), the damping is dominated by the forebody only and is conservatively modeled in the aerodatabase as a constant value. Therefore, the effective and peak torques are identical.

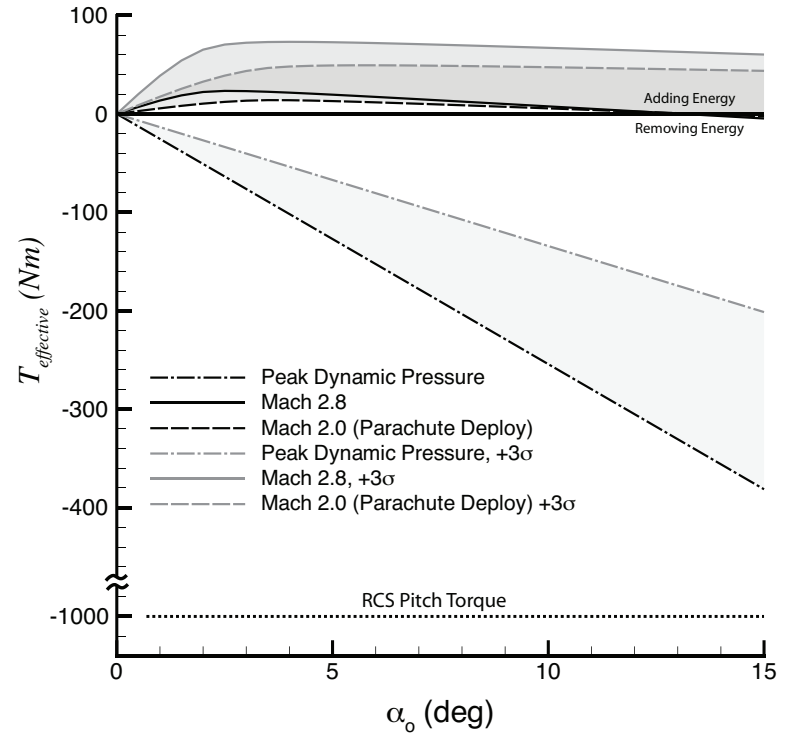

(a) Effective damping vs. amplitude

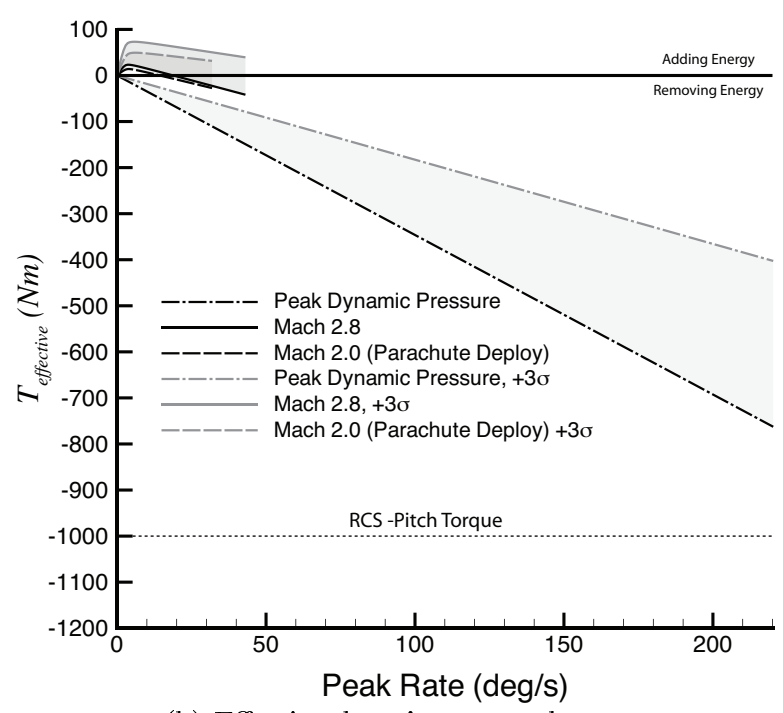

(b) Effective damping vs. peak rate

Figure 14. Effective damping torques based on AerospaceComputing BR data at several points along reference trajectory

\section{Conclusion}

A ballistic range test was performed to determine the dynamic stability model for the Mars Science Laboratory entry vehicle. Two independent data reduction efforts identified nonlinear models for pitch damping, $C_{m_{q}}$, as functions of angle-of-attack ( $\alpha$ from $0^{\circ}$ to $30^{\circ}$ ) and Mach number (Mach 1.5 to 3.5). While the functional forms identified by the two efforts look very different, they predict similar levels of dynamic stability when used in simulation. The agreement is closest in the Mach and angle-of-attack space anchored by the largest number of ballistic range data points. The largest disagreement occurs at small angles. This is not unexpected as it is very difficult to get small oscillation data in the ballistic range. At larger oscillation amplitudes, the effective damping of the two models are in very close agreement. The MSL pitch damping was found to be very similar to the Mars Exploration Rover pitch damping characteristics (determined with a similar ballistic range test). MSL may be slightly more dynamically stable than MER and no significant effects due to the change in backshell geometry were identified.

Lifting models cannot be safely tested at the Eglin AFB ballistic range, so all models tested were bodies of revolution with no cg offset. To account for lifting effects when the data is used in simulation, the peak instabilities are shifted to the current trim angle-of-attack. This approach is used for the MSL aerodatabase to be conservative. Further testing would be required to assess the true variation of pitch damping with radial cg offset.

The destabilizing effects of the pitch damping data were compared to the reaction control system of the MSL EV. Peak dynamic torques should never approach the torques generated by the RCS system along any part of the trajectory and the effective damping should act only as a slight perturbance to be easily countered by the controller. As the controller is continuously damping any excessive rates, the dynamic instabilities 
can not act on the vehicle uncorrected. No large oscillation amplitudes or rates are expected.

\section{References}

${ }^{1}$ Steinberg, S., "Experimental Pitch Damping Derivatives for Candidate Viking Entry Configurations at Mach Numbers from 0.6 Through 3.0," TR- 3709005, Martin Marietta Corporation, 1970.

${ }^{2}$ Teramoto, S., Hiraki, K., and Fugii, K., "Numerical Analysis of Dynamic Stability of a Reentry Capsule at Transonic Speeds," AIAA Journal, Vol. 39, No. 4, April 2001, pp. 646-653.

${ }^{3}$ Chapman, G. T., Mitcheltree, R. A., and Hathaway, W. H., "Transonic and Low Supersonic Static and Dyanmic Aerodynamic Characteristics of the Stardust Sample Return Capsule," AIAA 99-1021, 1999.

${ }^{4}$ Cheatwood, F., Winchenbach, G., Hathaway, W., and Chapman, G., "Dynamic Stability Testing of the Genesis Sample Return Capsule," AIAA 2000-1009, January 2000.

${ }^{5}$ Spencer, D. A., Blanchard, R. C., Braun, R. D., Kallemeyn, P. H., and Thurman, S. W., "Mars Pathfinder Entry, Descent, and Landing Reconstruction," Journal of Spacecraft and Rockets, Vol. 36, No. 3, May-June 1999, pp. 357-366.

${ }^{6}$ Steinberg, S., Uselton, B., and Siemers, P., "Viking Configuration Pitch Damping Derivatives as Influenced by Support Interference and Test Technique at Transonic and Supersonic Speeds," AIAA 72-1012, September 1972.

${ }^{7}$ Schoenenberger, M., Hathaway, W., Yates, L., and Desai, P., "Ballistic Range Testing of the Mars Exploration Rover Entry Capsule," AIAA 2005-0055, January 2005.

${ }^{8}$ Winchenbach, G. L., "Aerodynamic Testing in a Free-Flight Spark Range," Tech. Rep. WL-TR-1997-7006, Wright Laboratory, Armament Directorate, Weapon Flight Mechanics Division (WL/MNAV), Eglin AFB, FL, April 1997.

${ }^{9}$ Hathaway, W. H. and Whyte, R. H., "Aeroballistic Research Facility Free Flight Data Analysis Using Maximum Likelihood Method," AFATL-TR -79-98, Air Force Armament Laboratory, Eglin AFB, FL, December 1979.

${ }^{10}$ Yates, L. A., "A Comprehensive Aerodynamic Data Reduction System for Aeroballistic Ranges," WL-TR -96-7059, Wright Laboratory, Eglin AFB, FL, October 1996.

${ }^{11}$ Hathaway, W., "Aerodynamic Results: NASA - MSL Test Configuration," ARO 07-0924, Arrow Tech Associates for Aeroballistic Research Facility, Eglin AFB, September 2007.

${ }^{12}$ Fischer, M. A. and Hathaway, W. H., "ARFDAS Users Manual," AFATL-TR -88-48, Air Force Armament Laboratory, Eglin AFB, FL, November 1988.

${ }^{13}$ Yates, L. and Chapman, G. T., "Analysis of Data from Ballistic Range Tests of the Mars Science Laboratory (MSL) Vehicle," Tech. rep., Aerospace Computing Inc., Mountain View, CA, August, 2007.

${ }^{14}$ Owens, B. D. and Tomek, D., "18-CD Subsonic-Transonic Crew Module Dynamic Stability Test in the NASA LaRC Transonic Dynamics Tunnel," NASA/EG-CAP-07-99, February, 2009.

${ }^{15}$ Schoenenberger, M., Cheatwood, F., and Desai, P., "Static Aerodynamics of the Mars Exploration Rover Entry Capsule," AIAA 2005-0056, January 2005.

${ }^{16}$ Flaherty, T. M., "Aerodynamics Data Book," TR- 3709014, Martin Marietta Corporation, 1972.

${ }^{17}$ Redd, B., Olsen, D. M., and Barton, R. L., "Relationship Between the Aerodynamic Damping Derivatives Measured as a Function of Instantaneous Angular Displacement and the Aerodynamic Damping Derivatives Measured as a Function of Oscillation Amplitude," Tech. Rep. NASA TN D-2855, 1965.

${ }^{18}$ Schoenenberger, M. and Queen, E. M., "Limit Cycle Analysis Applied to the Oscillations of Decelerating Blunt-Body Entry Vehicles," NATO RTO-MP-AVT-152-006, 2008.

${ }^{19}$ Schoenenberger, M., Queen, E. M., and Litton, D., "Oscillation Amplitude Growth for a Decelerating Object with Constant Pitch Damping," AIAA 2006-6148, 2006. 